\title{
Beam-based model of broad-band impedance of the Diamond Light Source
}

\author{
Victor Smaluk, Ian Martin, and Richard Fielder \\ Diamond Light Source, Oxfordshire OX11 ODE, United Kingdom \\ Riccardo Bartolini \\ Diamond Light Source, Oxfordshire OX11 ODE, United Kingdom and John Adams Institute, \\ University of Oxford, Oxford OX1 3RH, United Kingdom \\ (Received 17 December 2014; published 23 June 2015)
}

\begin{abstract}
In an electron storage ring, the interaction between a single-bunch beam and a vacuum chamber impedance affects the beam parameters, which can be measured rather precisely. So we can develop beambased numerical models of longitudinal and transverse impedances. At the Diamond Light Source (DLS) to get the model parameters, a set of measured data has been used including current-dependent shift of betatron tunes and synchronous phase, chromatic damping rates, and bunch lengthening. A matlab code for multiparticle tracking has been developed. The tracking results and analytical estimations are quite consistent with the measured data. Since Diamond has the shortest natural bunch length among all light sources in standard operation, the studies of collective effects with short bunches are relevant to many facilities including next generation of light sources.
\end{abstract}

DOI: 10.1103/PhysRevSTAB.18.064401

PACS numbers: 29.20.-c, 29.27.Bd

\section{INTRODUCTION}

Collective effects of beam dynamics in particle accelerators result from the interaction of a particle beam with electromagnetic fields induced in a vacuum chamber by the beam itself. The most significant result of the collective effects is instability of longitudinal or transverse motion leading to deterioration of the beam quality or even to beam loss. Studies of collective effects are essential both for designing new accelerators and for understanding the beam dynamics in the already operated facilities in order to improve their performance.

In the theory of collective effects (see, for example, $[1,2])$, the interaction between the beam and wake fields is described using a concept of a wake function, which is determined as a normalized integral of the Lorentz force that acts on a test particle moving behind a leading one exciting the wake fields. The wake function is a timedomain response of a vacuum chamber to the excitation by a point charge. In most practical cases, it is enough to consider only monopole longitudinal and dipole transverse wake functions to analyze stability of the beam motion. In the ultrarelativistic case, the wake function is determined by the geometry and electromagnetic properties of the chamber only, and it is independent of the charge distribution in the beam. In the frequency domain, the beam-wake interaction is characterized by frequency-dependent

\footnotetext{
*victor.smalyuk@diamond.ac.uk

Published by the American Physical Society under the terms of the Creative Commons Attribution 3.0 License. Further distribution of this work must maintain attribution to the author $(s)$ and the published article's title, journal citation, and DOI.
}

coupling impedances, which are Fourier transforms of the corresponding wake functions.

There is a limited number of analytical formulas to calculate impedances of simplest vacuum chamber sections, such as pill-box cavities or step transitions. Real vacuum chambers usually have a complex geometry including collimators, transitions between various crosssections, button electrodes, striplines, etc. Practically, for estimation of wake functions and impedances of a vacuum chamber section, finite-difference simulation codes are used (e.g., GdfidL [3] and CST Particle Studio [4]). The problem is that the wake function is not calculated directly, the simulation code output is a wake potential which is a convolution of the wake function with longitudinal bunch profile. So the bandwidth of the impedance derived from the simulated wake potential is limited by the bunch spectrum width which is inversely proportional to the bunch length. For a bunch length of a few millimeters, full 3D simulation of wake fields in a big and complex structure is quite difficult too, because huge memory and processor time are required. Generally, there is no way to derive a point-charge wake function from a wake potential of a finite-length bunch, although asymptotic models for some geometries have been developed [5].

Calculation of impedance budget is an essential stage of a new accelerator design. If the interference of the wake fields induced by the beam in various components of the vacuum chamber is negligible (large distance between the components or fast-damping wake fields), the impedances are additive at any frequencies. In this case, the impedance of the entire vacuum chamber can be represented as a sum of impedances of its components. A total frequencydependent impedance is usually very complex and hardly 
suitable for beam stability analysis. A simplified model characterized by very few parameters such as total loss factor and inductance (or normalized impedance) for the longitudinal wake, and horizontal/vertical kick factors for the transverse wakes, are generally used to characterize the beam-chamber interaction, see some recent results in [6-8].

For the Diamond Light Source, we have developed a model consisting of a broad-band resonator (BBR) and restive-wall impedance. The latter is calculated analytically using known dimensions and resistivity of the vacuum chamber. Neither constant resistance nor inductance were needed to fit the measured data. Shunt impedance and resonant frequency values of the model BBR have been obtained from beam-based measurements, the quality factor is assumed equal to 1 . To improve the fit accuracy of the measured data, a combined fitting technique was developed and realized. For the longitudinal BBR, both current-dependent bunch lengthening and synchronous phase shift were fit with two parameters, shunt impedance $R_{\|}$and resonant frequency $\omega_{\|}$. For the horizontal or vertical BBR, both current-dependent betatron tune shift and chromatic damping rate were fit with two parameters, shunt impedance $R_{x, y}$ and resonant frequency $\omega_{x, y}$.

\section{RESISTIVE-WALL AND BROAD-BAND RESONATOR IMPEDANCE}

Total impedance of a vacuum chamber includes geometric and resistive-wall components. Vacuum chambers of modern electron storage rings are made with rather small apertures (especially vertical), so the resistive-wall impedance can be comparable with the geometric one. The Diamond Light Source has a $2 \mathrm{~mm}$ thick stainless-steel vacuum chamber, the in-vacuum narrow-gap $(5 \mathrm{~mm}$ if closed) insertion devices (IDs) are covered by coppernickel foil $(50 \mu \mathrm{m} \mathrm{Cu})$. Formulas to calculate the resistivewall impedance have been derived analytically for the elliptic and rectangular vacuum chambers [9]. If the wall thickness is much larger than the skin depth

$$
\delta_{s}(\omega)=\sqrt{\frac{2 c}{\mu_{r} \sigma_{c} Z_{0}|\omega|}},
$$

the longitudinal $Z_{\|} / L$ and transverse $Z_{x} / L, Z_{y} / L$ impedances per unit length are

$$
\begin{gathered}
\frac{Z_{\|}^{\mathrm{rw}}(\omega)}{L}=(1+i \operatorname{sign} \omega) \frac{Z_{0}|\omega| \delta_{s}(\omega)}{4 \pi c b} G_{0}, \\
\frac{Z_{x, y}^{\mathrm{rw}}(\omega)}{L}=(\operatorname{sign} \omega+i) \frac{Z_{0} \delta_{s}(\omega)}{2 \pi b^{3}} G_{1 x, y},
\end{gathered}
$$

where $c$ is the speed of light, $\mu_{r}$ and $\sigma_{c}$ are the relative permeability and conductivity of the chamber material, respectively; $Z_{0}$ is the free space impedance; $b$ is the half-aperture. Assuming cutoff frequency of the vacuum chamber about $10 \mathrm{GHz}$, the skin depth is about $4 \mu \mathrm{m}$ in stainless steel and is about $0.5 \mu \mathrm{m}$ in copper. These values are much smaller than the vacuum chamber thickness (2 $\mathrm{mm}$ ) and formulas (2), (3) are valid for the frequencies much smaller than $c / \sqrt{\delta_{s} b} \simeq 2 \pi \cdot 200 \mathrm{GHz}$. Frequency dependence of the resistive-wall impedance is discussed in [10] for the frequency range of $1-10^{14} \mathrm{~Hz}$.

The form-factors $G_{0}, G_{1 x}$, and $G_{1 y}$ are determined by the vacuum chamber cross section. For an elliptic chamber with horizontal $a$ and vertical $b$ semi-axes, the form-factors are [9]:

$$
\begin{aligned}
G_{0}\left(u_{0}\right) & =\frac{\sinh u_{0}}{2 \pi} \int_{0}^{2 \pi} \frac{Q_{0}^{2}(v)}{\sqrt{\sinh ^{2} u_{0}+\sin ^{2} v}} d v, \\
G_{1 x, y}\left(u_{0}\right) & =\frac{\sinh ^{3} u_{0}}{4 \pi} \int_{0}^{2 \pi} \frac{Q_{1 x, y}^{2}(v)}{\sqrt{\sinh ^{2} u_{0}+\sin ^{2} v}} d v,
\end{aligned}
$$

where

$$
\begin{gathered}
Q_{0}(v)=1+2 \sum_{m=1}^{\infty}(-1)^{m} \frac{\cos 2 m v}{\cosh 2 m u_{0}}, \\
Q_{1 x}(v)=2 \sum_{m=0}^{\infty}(-1)^{m}(2 m+1) \frac{\cos (2 m+1) v}{\cosh (2 m+1) u_{0}}, \\
Q_{1 y}(v)=2 \sum_{m=0}^{\infty}(-1)^{m}(2 m+1) \frac{\sin (2 m+1) v}{\sinh (2 m+1) u_{0}},
\end{gathered}
$$

and

$$
u_{0}=\operatorname{arccosh} \frac{1}{\sqrt{1-(b / a)^{2}}}
$$

The vacuum chamber of the Diamond Light Source has average horizontal half-aperture $a=40 \mathrm{~mm}$ and vertical half-aperture $b=12 \mathrm{~mm}$, so $G_{0}=0.98, G_{1 x}=0.43$, $G_{1 y}=0.83$.

The geometric impedance of any vacuum chamber section can be approximately represented as a sum of equivalent resonators, each of them contributes the impedance:

$$
\begin{gathered}
Z_{\|}(\omega)=\frac{R_{\|}}{1+i Q\left(\frac{\omega}{\omega_{\|}}-\frac{\omega_{\|}}{\omega}\right)}, \\
Z_{x, y}(\omega)=\frac{\omega_{x, y}}{\omega} \frac{R_{x, y}}{1+i Q\left(\frac{\omega}{\omega_{x, y}}-\frac{\omega_{x, y}}{\omega}\right)},
\end{gathered}
$$

where $R_{\|}, R_{x, y}$ are the shunt impedances, $\omega_{\|}, \omega_{x, y}$ are the resonance frequencies, and $Q$ is the quality factor. The broad-band (low-Q) impedance causing intra-bunch 
particle interaction can be assumed to be additive, because the wake fields dissipate rapidly, and effects of superposition of the wake fields excited by the same bunch in different components of the vacuum chamber are negligible. To characterize the single-bunch collective effects in many important practical cases, the total broad-band impedance of the vacuum chamber can be approximated by a single broad-band resonator (9), (10) assuming $Q \simeq 1$.

\section{LONGITUDINAL IMPEDANCE}

Interaction of a bunched beam with short-range longitudinal wake fields (broad-band impedance) deforms the longitudinal bunch profile $\lambda(t)$, which is Gaussian for a zero-intensity bunch:

$$
\lambda_{0}(t)=\frac{1}{\sqrt{2 \pi} \sigma_{0}} \exp \left(-\frac{t^{2}}{2 \sigma_{0}^{2}}\right),
$$

with the r.m.s. bunch length (in seconds)

$$
\sigma_{0}=\frac{\alpha}{\omega_{s}} \frac{\sigma_{E}}{E},
$$

where $\alpha$ is the momentum compaction factor, $\omega_{s}$ is the synchrotron frequency, $E$ is the beam energy, $\sigma_{E} / E$ is the relative energy spread. At small beam currents, the energy spread of the relativistic electron beam is independent of its intensity and $\lambda(t)$ as a function of the average bunch current $I_{b}$ can be approximately described by the Haissinski integral equation [11]:

$$
\lambda(t)=K \lambda_{0}(t) \exp \left[-\frac{\alpha I_{b}}{\omega_{s}^{2} \sigma_{0}^{2} E / e} \int_{-\infty}^{t} S(t+\tau) \lambda(\tau) \mathrm{d} \tau\right] .
$$

The beam-impedance interaction is described by the stepfunction wake potential

$$
S(t)=\int_{0}^{t} W_{\|}(\tau) \mathrm{d} \tau,
$$

$W_{\|}$is the longitudinal wake function. Normalizing factor $K$ is determined by the condition $\int_{-\infty}^{\infty} \lambda(t) \mathrm{d} t=1$. For a positive momentum compaction $\alpha$, the intensity-dependent deformation of the longitudinal bunch profile $\lambda(t)$ causes the bunch lengthening, which can be approximately described by a cubic equation using a concept of effective impedance [12].

The bunch profile has been measured using a streakcamera [13] for the single-bunch beam current $I_{b}$ varied in the range of $0.05-0.8 \mathrm{~mA}$ with the following parameters of the Diamond storage ring: $E=3 \mathrm{GeV}, \sigma_{0}=12.5 \mathrm{ps}$, $\alpha=1.66 \times 10^{-4} ; \omega_{s}=\nu_{s} \omega_{0}, \nu_{s}=0.0042$ is the synchrotron tune, $\omega_{0}=2 \pi f_{0}, f_{0}=533.8 \mathrm{kHz}$ is the revolution frequency. Figure 1 shows three examples of the bunch profiles measured at $0.05 \mathrm{~mA}, 0.2 \mathrm{~mA}$, and $0.5 \mathrm{~mA}$ together with the fit curves obtained by numerical solution of the Haissinski equation (13), the wake function $W_{\|}$ includes both BBR and resistive-wall terms.

The beam-impedance interaction results also in coherent loss of the bunch energy, which is proportional to the square of bunch charge $q_{b}$. The proportionality factor is the loss factor

$$
k_{\|}=\frac{1}{2 \pi} \int_{-\infty}^{\infty} Z_{\|}(\omega) h(\omega) \mathrm{d} \omega
$$

where $h(\omega)=\Lambda(\omega) \Lambda^{*}(\omega)$ is the bunch power spectrum, $\Lambda(\omega)$ is the Fourier transform of the longitudinal beam density distribution $\lambda(t)$. For a Gaussian beam (11), $h(\omega)=e^{-\omega^{2} \sigma_{0}^{2}}$.

The coherent energy loss is compensated in the accelerating rf cavities every beam turn, as well as the energy loss $U$ caused by synchrotron radiation. The loss factor can be derived from the energy balance of the bunch:

$$
q_{b} V_{\mathrm{rf}} \sin \left(\phi_{s}+\Delta \phi_{s}\right)=U+k_{\|} q_{b}^{2},
$$

where $V_{\mathrm{rf}}$ is the rf voltage, $\phi_{s}$ is the synchronous phase. If the phase shift $\Delta \phi_{s}$ is small, it can be assumed proportional to the beam current $I_{b}=q_{b} f_{0}$ and the loss factor $k_{\|}$:

$$
\Delta \phi_{s}=\frac{I_{b} k_{\|}}{f_{0} V_{\mathrm{rf}} \cos \phi_{s}},
$$

Rewriting Eq. (16) in the form:

$$
k_{\|}=\frac{\nu_{s}^{2} \omega_{0}^{2} E / e}{\alpha} \frac{\Delta t_{s}}{\Delta I_{b}}, \quad \Delta t_{s}=\frac{\Delta \phi_{s}}{\omega_{\mathrm{rf}}},
$$

we can estimate the loss factor from the measurable beam parameters: current-dependent shift of the bunch synchronous time $\Delta t_{s} / \Delta I_{b}$ and the synchrotron tune $\nu_{s}$. The revolution frequency $\omega_{0}$ and the rf frequency $\omega_{\mathrm{rf}}$ are known with high precision.

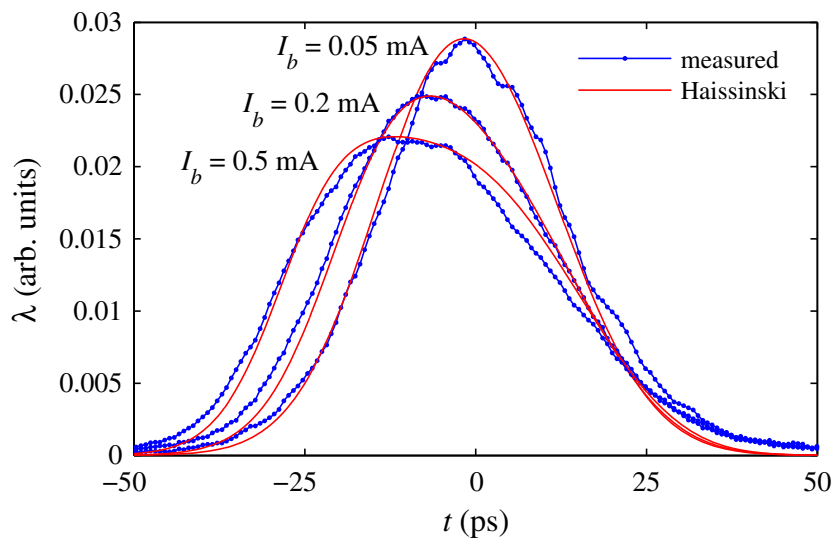

FIG. 1. Measured longitudinal beam profile with Haissinski equation fit. 
A multiparticle tracking code has been developed to study the single-bunch beam stability. For the longitudinal motion, the numerical model includes collective effects and nonlinearity of the rf potential well. The beam is represented as an ensemble of macroparticles with Gaussian initial distribution. One-turn transformations of the longitudinal position $z_{i}$ and relative energy deviation $\varepsilon_{i}=\Delta E / E$ of $i$ th macroparticle are

$$
\begin{aligned}
\varepsilon_{i, n+1} & =\varepsilon_{i, n}+\frac{V_{\mathrm{rf}}}{E / e} \cos \left(\omega_{\mathrm{rf}} \frac{z_{i, n}}{c}-\phi_{s}+\frac{\pi}{2}\right)-\frac{U}{E}, \\
z_{i, n+1} & =z_{i, n}-\alpha c T_{0} \varepsilon_{i, n+1},
\end{aligned}
$$

$T_{0}=1.873 \mu \mathrm{s}$ is the revolution period. Then radiation damping $-\left(2 T_{0} / \tau_{s}\right) \varepsilon_{n+1}$ and quantum excitation $\left(2 \sigma_{E} / E\right) \sqrt{T_{0} / \tau_{s}}$ terms are also added to $\varepsilon_{n+1}$.

To simulate the beam-impedance interaction, each macroparticle gets an energy kick $\Delta \varepsilon_{i}$ every turn:

$$
\Delta \varepsilon_{i}=-V_{\|}\left(t_{i}\right) \frac{q_{p}}{E / e}
$$

where $q_{p}$ is the macroparticle charge, $t_{i}=z_{i} / c$,

$$
V_{\|}(t)=\frac{1}{2 \pi} \int_{-\infty}^{\infty} Z_{\|}(\omega) \Lambda(\omega) e^{i \omega t} d \omega
$$

$Z_{\|}(\omega)$ is the total longitudinal impedance including BBR and resistive-wall ones.

The bunch length and synchronous time have been measured using a streak-camera [13] as functions of the bunch current $I_{b}$ varied in the range of $0.05-0.8 \mathrm{~mA}$. The parameters of the Diamond storage ring related to the measurements are: $E=3 \mathrm{GeV}, \sigma_{E} / E=1.1 \times 10^{-3}$, $f_{0}=533.8 \mathrm{kHz}, \quad \alpha=1.66 \times 10^{-4}, \quad \nu_{s}=0.0042, \quad \sigma_{0}=$ $12.5 \mathrm{ps}\left(V_{\mathrm{rf}}=2.5 \mathrm{MV}\right.$, wigglers are switched on).

The measured bunch profiles have been approximated by numerical solutions of Haisinski equation (13); at the same time, the synchronous phase shift has been approximated by Eq. (16). The longitudinal impedance $Z_{\|}$is a sum of the geometric impedance and the resistive-wall one. The resistive-wall impedance (2) is calculated for an elliptic stainless steel chamber $\left(\mu_{r}=1\right.$ and $\left.\sigma_{c}^{-1}=7.3 \times 10^{-7} \Omega \mathrm{m}\right)$ with average half-apertures $a=40 \mathrm{~mm}$ and $b=12 \mathrm{~mm}$. The geometric impedance is modeled by a single broadband $(Q=1)$ resonator $(9)$, the resonance frequency $\omega_{\|}$ and shunt impedance $R_{\|}$are the fit parameters. The best fit gives $\omega_{\|}=2 \pi \cdot 22 \mathrm{GHz}, R_{\|}=8 \mathrm{k} \Omega$, average fit error is about $12 \%$.

Figure 2 shows the model of longitudinal impedance including the BBR (9) and the resistive-wall (2) ones. A zero-current loss factor calculated with formula (14) using the model impedance and Gaussian bunch with

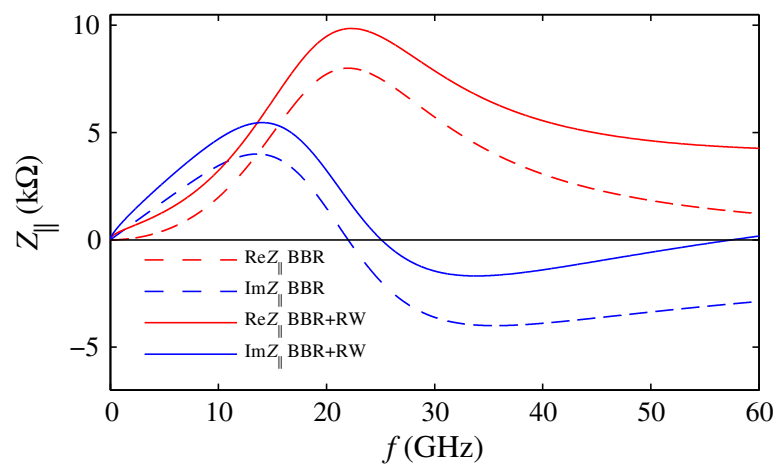

FIG. 2. Longitudinal impedance model (BBR + resistive wall).

$\sigma_{t}=12.5 \mathrm{ps}$ is $54 \mathrm{~V} / \mathrm{pC}$, whereas the loss factor estimated from the measured data is $58 \pm 6 \mathrm{~V} / \mathrm{pC}$.

The model impedance shown in Fig. 2 was used for multiparticle tracking. The machine parameters used for the tracking are the same as they were during the beam measurements, the number of macroparticles is $5 \times 10^{5}$. Figure 3 shows the bunch full width at half-maximum (FWHM) as a function of beam current (upper plot) and the current-dependent shift of the bunch synchronous time (lower plot). The measured data are presented by dots with error bars together with the fit curves (red lines) and with the tracking results (green lines).

As one can see, the measured data are in agreement with the formula within the statistical accuracy of the measurement. The bunch lengthening calculated by multiparticle tracking agrees quite well too with both the measurement

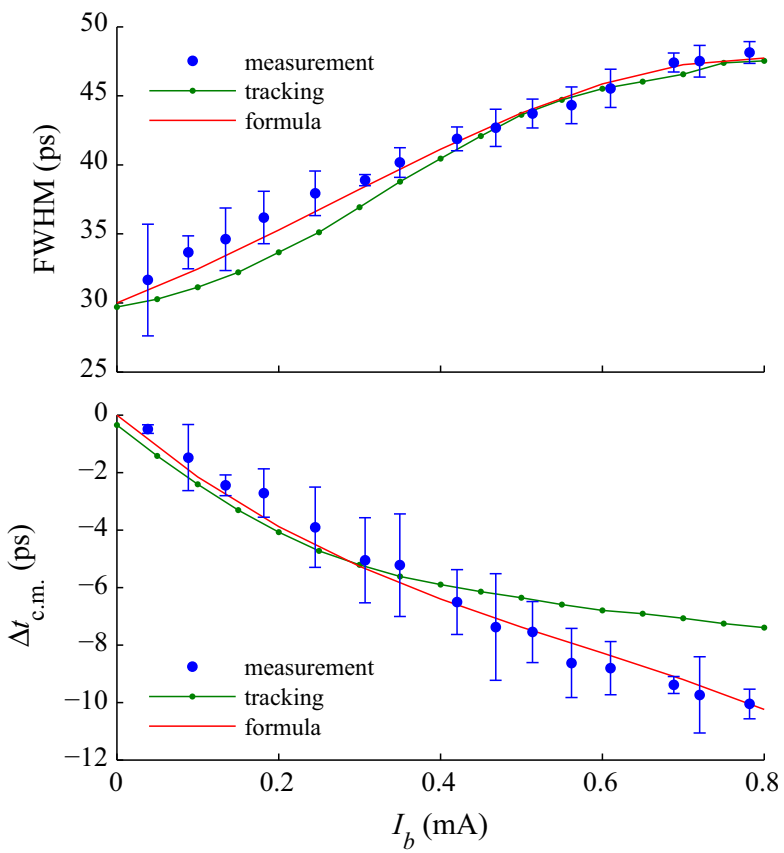

FIG. 3. Current-dependent bunch lengthening (upper plot) and synchronous time shift (lower plot). Measurement, formula, and tracking. 
and the formula. For a higher beam current, the synchronous time shift calculated by tracking is underestimated in comparison with the others two. This discrepancy can probably result from the constant synchronous phase (18) used in the tracking code, whereas the real rf phase is autotuned with the current-dependent beam load.

\section{TRANSVERSE IMPEDANCE}

Resonance interaction of the beam with short-range transverse wake fields characterized by the transverse broad-band impedance, results in the transverse mode coupling. The wake fields induced by the head of a bunched beam act on particles of its tail part (a head-tail effect). The head and tail of the bunch exchange places periodically due to synchrotron oscillations. The beam motion is dependent on the chromaticity $\xi \equiv \frac{d \nu_{\beta}}{d E / E}, \nu_{\beta}$ is the betatron tune, $d E / E$ is the relative energy deviation.

If the chromaticity is zero, a fast head-tail instability occurs when the beam current exceeds a certain threshold, causing an exponential growth of betatron oscillations. As a result, the beam particles are lost on the aperture and the beam intensity drops down to the threshold value. In the frequency domain, the instability arises when the coherent (center-of-mass) mode is coupled with lower head-tail mode. The current-dependent frequency shift of the coherent mode almost reaches the synchrotron frequency at the threshold.

If the chromaticity is nonzero, a chromatic head-tail effect arises and some head-tail modes become unstable for any beam current, without a threshold. The coherent (center-of-mass) mode damps upon the positive chromaticity and becomes unstable when the latter is negative, and the higher-order head-tail modes behave oppositely. The rising/damping rates decrease rapidly with the mode number, and the higher-order modes are usually not dangerous for beam stability, since they are suppressed by the radiation damping. Since only a few of the lowest modes are essential, the eigenmode analysis is quite efficient for study of the head-tail effect. The complex frequency $\Omega=\omega+i / \tau$ of $l$ th head-tail mode can be found solving the eigenvalue problem [1]

$$
\operatorname{det}\left[\left(\frac{\Omega-\omega_{\beta}}{\omega_{s}}-l\right) \mathbf{I}-\mathbf{M}\right]=0,
$$

here $\omega_{\beta}$ is the unperturbed betatron frequency, $\tau$ is the rising/damping time. The matrix elements are

$$
M_{k k^{\prime}}=I_{b} \frac{\beta}{2 \nu_{s} E / e} \sum_{p=-\infty}^{\infty} Z_{\perp}\left(\omega^{\prime}\right) g_{l k}\left(\omega^{\prime}-\omega_{\xi}\right) g_{l k^{\prime}}\left(\omega^{\prime}-\omega_{\xi}\right),
$$

where $\beta$ is the average beta function, $\omega^{\prime}=p \omega_{0}+\omega_{\beta}+$ $l \omega_{s}, \omega_{\xi}=\xi \omega_{0} / \alpha$ is the chromatic frequency. The functions characterizing oscillation modes of the Gaussian bunch are

$$
g_{l k}(\omega)=\frac{1}{\sqrt{2 \pi k !(|l|+k) !}}\left(\frac{\omega \sigma_{t}}{\sqrt{2}}\right)^{|l|+2 k} \exp \left(-\frac{\omega^{2} \sigma_{t}^{2}}{2}\right) .
$$

If the frequency shift of coherent (0th) mode is small compared with the synchrotron frequency $\omega_{s}$, the linear approximation [1] is applicable:

$$
\frac{d \Omega}{d I_{b}}=i \frac{\beta Z_{\perp}^{\mathrm{eff}}}{4 \sqrt{\pi} \sigma_{t} E / e}
$$

where

$$
Z_{\perp}^{\mathrm{eff}}=\frac{\sum_{p=-\infty}^{\infty} Z_{\perp}\left(\omega^{\prime}\right) h\left(\omega^{\prime}-\omega_{\xi}\right)}{\sum_{p=-\infty}^{\infty} h\left(\omega^{\prime}-\omega_{\xi}\right)}
$$

is the effective broad-band impedance, $h(\omega)$ is the bunch power spectrum.

If the chromaticity is positive, the frequency shift $\Delta \omega=$ $\operatorname{Re} \Omega-\omega_{\beta}$ and chromatic damping time $\tau=1 / \operatorname{Im} \Omega$ of the coherent $(l=0)$ mode can be obtained by spectral analysis of beam oscillations registered by a turn-by-turn beam position monitor.

Computer simulations of the interaction between a single-bunch beam and short-range transverse wake fields have been performed using a multiparticle tracking code. For the transverse motion, the numerical model includes linear matrix transformations with chromatic shift of betatron tunes as well as collective effects. Betatron motion of $i$ th macroparticle at $n$th turn is described by the one-turn matrix transformation of a position-angle vector

$\left[\begin{array}{c}x_{i, n+1} \\ x_{i, n+1}^{\prime}\end{array}\right]=\left[\begin{array}{cc}\cos 2 \pi \nu_{i, n} & \beta \sin 2 \pi \nu_{i, n} \\ -\frac{1}{\beta} \sin 2 \pi \nu_{i, n} & \cos 2 \pi \nu_{i, n}\end{array}\right]\left[\begin{array}{c}x_{i, n} \\ x_{i, n}^{\prime}\end{array}\right]$.

Here $x$ and $x^{\prime}$ denotes horizontal or vertical position and angle, respectively; $\beta$ is the beta function, $\nu_{i, n}$ is the oneturn betatron phase advance including chromatic shift:

$$
\nu_{i, n}=\nu_{\beta}+\xi \varepsilon_{i, n}
$$

$\nu_{\beta}$ is the unperturbed betatron tune, $\varepsilon=\Delta E / E$ is the relative energy deviation, $\xi$ is the chromaticity. For the horizontal motion, the dispersive terms $\eta_{x}\left(1-\cos 2 \pi \nu_{i, n}\right)$ and $\frac{\eta_{x}}{\beta} \sin 2 \pi \nu_{i, n}$ are added to $x_{i, n+1}$ and $x_{i, n+1}^{\prime}$, respectively. Here $\eta_{x}$ is the dispersion. The radiation damping and quantum excitation terms are included as well.

To simulate the beam-impedance interaction, each macroparticle gets a transverse $\Delta x_{i}^{\prime}$ kick

$$
\Delta x_{i}^{\prime}=x V_{x}\left(t_{i}\right) \frac{q_{p}}{E / e},
$$

where $q_{p}$ is the macroparticle charge, 


$$
x V_{x}(t)=-\frac{i}{2 \pi} \int_{-\infty}^{\infty} Z_{x}(\omega) \Lambda_{x}(\omega) e^{i \omega t} d \omega
$$

$Z_{x}(\omega)$ is the broad-band transverse impedance (horizontal or vertical),

$$
\Lambda_{x}(\omega)=\int_{-\infty}^{\infty} \lambda(t) x(t) e^{-i \omega t} d t
$$

$\lambda(t)$ is the longitudinal bunch profile, $x(t)$ is the transverse position of corresponding particles.

A series of beam studies has been carried out at the Diamond storage ring in single-bunch mode and in two regimes: with 13 in-vacuum IDs open or closed. The chromaticity was set close to $\xi_{x}=\xi_{y}=0,1,2$. Both horizontal and vertical coherent beam oscillations were excited by the kickers. To measure turn-by-turn beam positions, the pickup of transverse multibunch feedback system [14] was used. Its high sensitivity allows us to limit the beam oscillation amplitude down to $100 \mu \mathrm{m}$ and thereby to reduce amplitude-dependent nonlinear effects, which can deteriorate the measurement accuracy. The measurements have been performed with the single-bunch current varying in the $0.05-0.8 \mathrm{~mA}$ range.

In Fig. 4, an example of the horizontal (upper plots) and vertical (lower plots) turn-by-turn beam position (left) measured with the bunch current of $0.03 \mathrm{~mA}, 0.17 \mathrm{~mA}$, and $0.6 \mathrm{~mA}$ and their spectra (right) are presented. The beam positions are plotted as functions of time, amplitude spectra-as functions of tune. Both horizontal and vertical chromaticities were set to 1 . As one can see, the damping time of the coherent oscillation mode decreases rapidly with the beam current. In the spectrum graph, it can be also seen that for $I_{b}=0.6 \mathrm{~mA}$, the amplitude of $-1 \mathrm{st}$
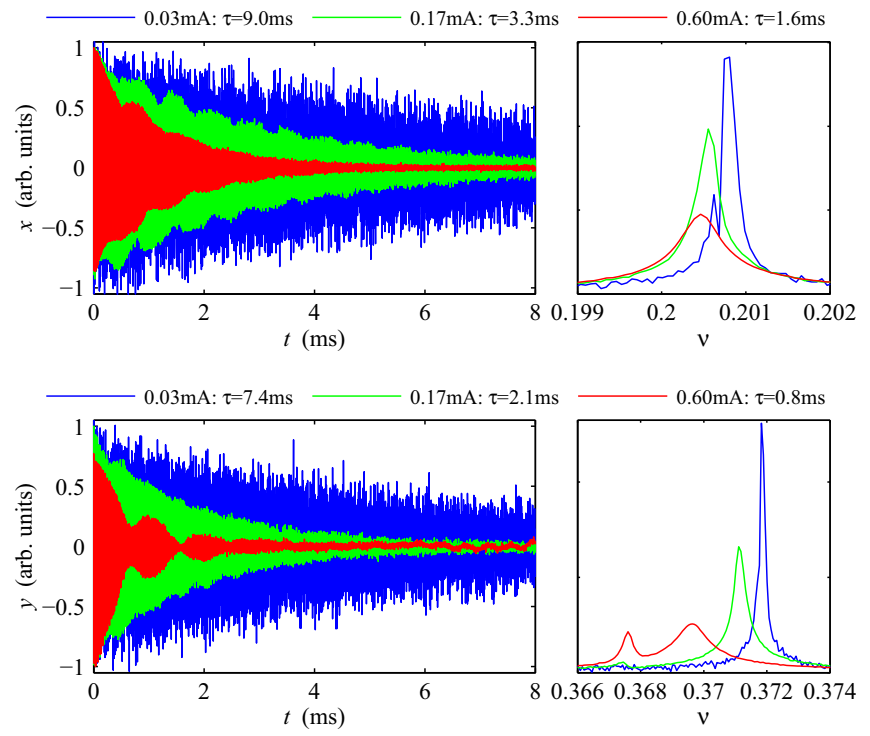

FIG. 4. Horizontal (upper plots) and vertical (lower plots) turnby-turn beam position (left) and spectra (right). vertical head-tail mode is almost the same as the amplitude of 0th mode, this indicates the proximity of the instability threshold.

The solutions of eigenvalue problem (20) have been used for approximation of the measured frequency shift (real part of $\Delta \Omega$ ) and chromatic damping rate (imaginary part of $\Delta \Omega$ ). This combined fitting technique allows us to improve the fit accuracy of the measured data, especially for the horizontal plane where the current-dependent tune shift is hardly measurable, whereas the damping rate can be measured with much better accuracy.

The total transverse model impedance consists of BBR and resistive-wall components. The resistive-wall impedance is calculated with formula (3) for a stainless steel chamber $\left(\mu_{r}=1\right.$ and $\left.\sigma_{c}^{-1}=7.3 \times 10^{-7} \Omega \mathrm{m}\right)$ with average horizontal half-aperture of $40 \mathrm{~mm}$ and vertical half-aperture of $13 \mathrm{~mm}$ (open IDs) and $12 \mathrm{~mm}$ (closed IDs). For both horizontal and vertical planes, the geometric impedance is modeled by a single broad-band $(Q=1)$ resonator (10), the resonance frequency $\omega_{x, y}$ and shunt impedance $R_{x, y}$ were the fit parameters.

The combined fit of the measured vertical currentdependent tune shift and chromatic damping rate gives the parameters of the vertical BBR impedance: the shunt impedance is $R_{y}=0.27 \mathrm{M} \Omega / \mathrm{m}$ if the IDs are open and $R_{y}=0.32 \mathrm{M} \Omega / \mathrm{m}$ if the IDs are closed, the resonant frequency is $\omega_{r y}=2 \pi \cdot 30 \mathrm{GHz}$ in both cases. Average fit error is about $10 \%$. Figure 5 shows the model of vertical broad-band impedance including the BBR (10) and the resistive-wall (3) components.

The model vertical impedance shown in Fig. 5 was used for multiparticle tracking. The machine parameters used for the tracking are the same as they were during the beam measurements, the number of macroparticles is $5 \times 10^{5}$. Figure 6 shows the current-dependent shift of vertical betatron tune $\nu_{y}=\omega_{y} / \omega_{0}$ (upper plot) and damping rate $1 / \tau_{y}$ (lower plot), the in-vacuum IDs are open. The measured values are shown by blue points with error bars, red lines represent the eigenvalues of (20) calculated

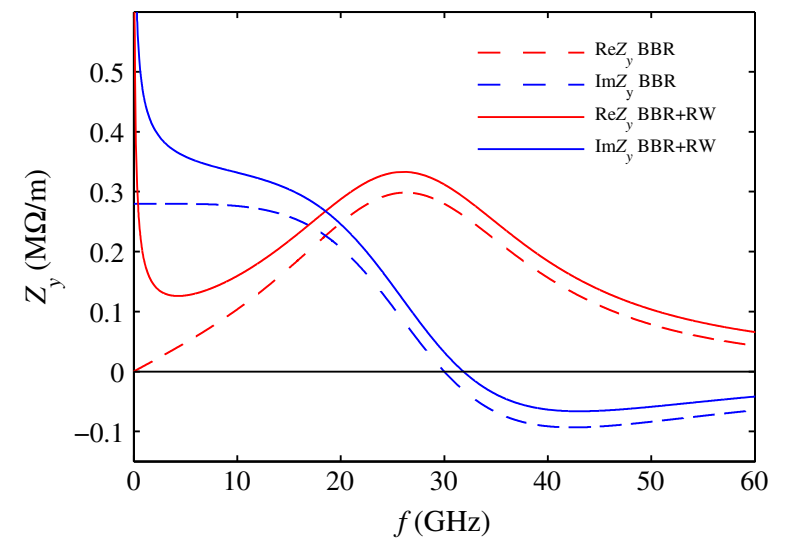

FIG. 5. Vertical impedance model (BBR + resistive wall). 

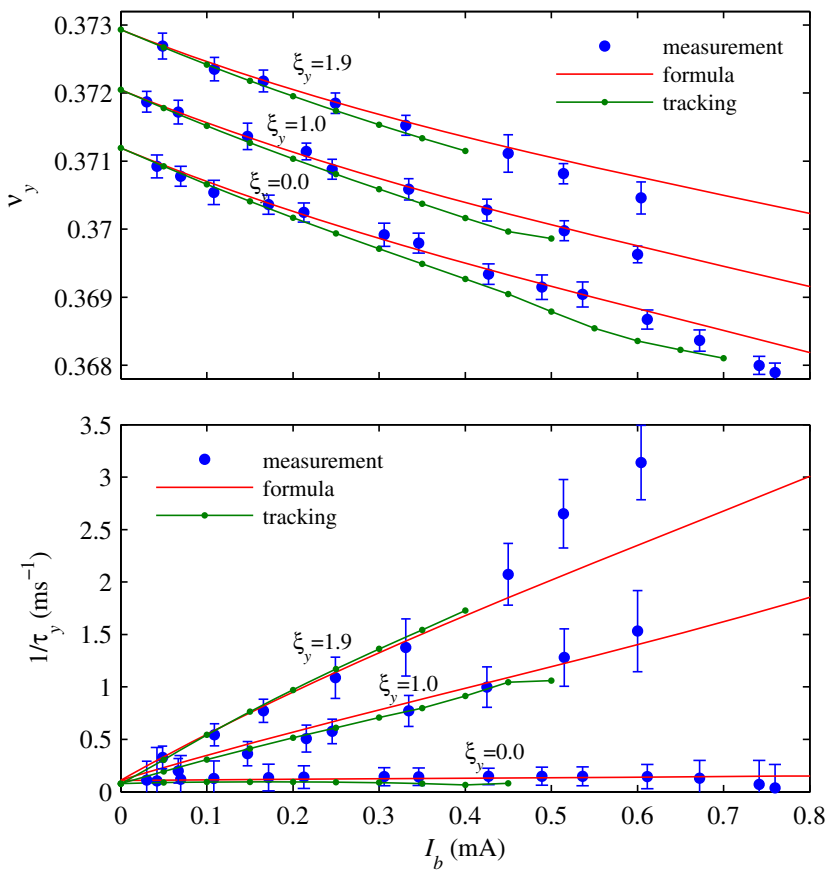

FIG. 6. Vertical betatron tune $\nu_{y}$ (upper plot) and damping rate $1 / \tau_{y}$ (lower plot) vs bunch current $I_{b}$. Measurement, formula, and tracking.

for 3 values of chromaticity according to the measurements, the green lines represent the tracking results. Note that the betatron tune graphs corresponding to different values of chromaticity are manually separated for better visibility in this and the next figures. A zero-current vertical kick factor calculated using the model broad-band impedance and Gaussian bunch with $\sigma_{t}=12.5 \mathrm{ps}$ is about $8 \mathrm{kV} /(\mathrm{pCm})$ if the in-vacuum IDs are open, and it is about $10 \mathrm{kV} /(\mathrm{pC} \mathrm{m})$ if the IDs are closed.

The same fit procedure has been performed for the measured horizontal current-dependent tune shift and chromatic damping rate. The fit results are the parameters of horizontal BBR impedance: $\omega_{r x}=2 \pi \cdot 16 \mathrm{GHz}$, $R_{x}=0.10 \mathrm{M} \Omega / \mathrm{m}, Q=1$. Possible variation related to

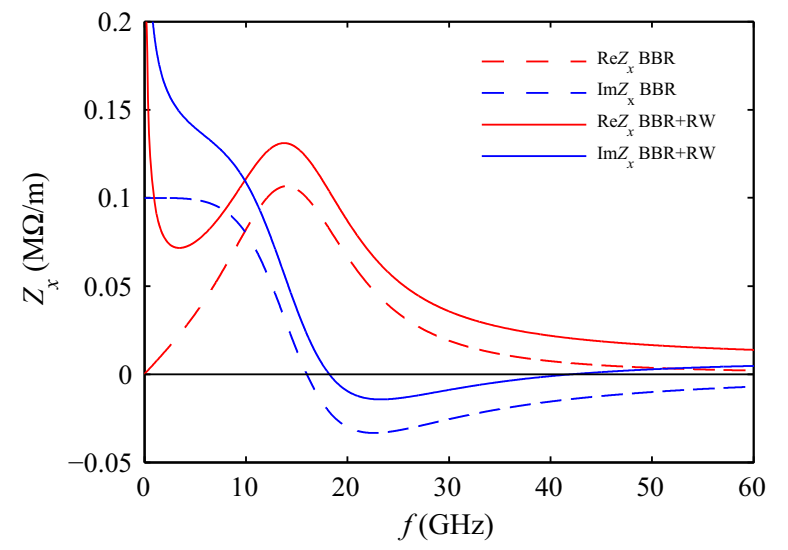

FIG. 7. Horizontal impedance model (BBR + resistive wall).
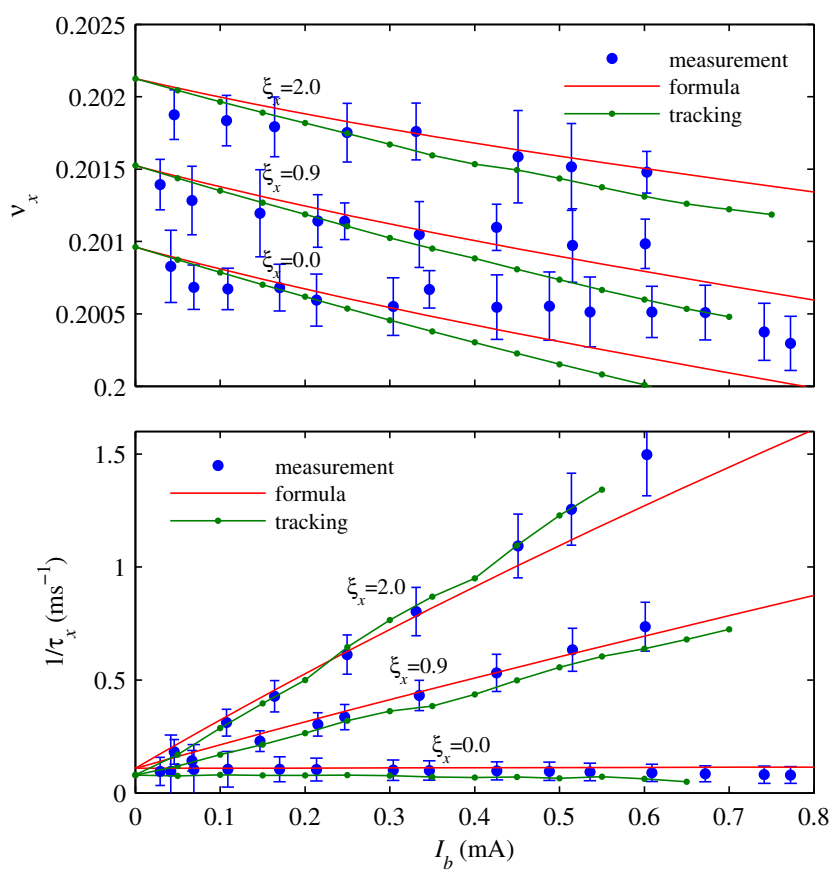

FIG. 8. Horizontal betatron tune $\nu_{x}$ (upper plot) and damping rate $1 / \tau_{x}$ (lower plot) vs bunch current $I_{b}$. Measurement, formula, and tracking.

the open IDs/closed IDs regimes was not detected in these measurements. Figure 7 shows the model of horizontal broad-band impedance including BBR and resistive-wall components. A zero-current horizontal kick factor calculated using the model impedance and Gaussian bunch with $\sigma_{t}=12.5 \mathrm{ps}$ is about $3 \mathrm{kV} /(\mathrm{pC} \mathrm{m})$.

Figure 8 shows the horizontal tune $\nu_{x}=\omega_{x} / \omega_{0}$ (upper plot) and damping rate $1 / \tau_{x}$ (lower plot) as functions of beam current. For the horizontal chromaticity of $0,1,2$, there are measured values (blue points with error bars), solutions of the eigenvalue problem (red lines), and the results of tracking of $5 \times 10^{5}$ macroparticles (green lines). As one can see, the coherent shift of the horizontal tune is very small because the dipole and quadrupole impedances contributed by asymmetric sections of vacuum chamber almost compensate each other, but the chromatic damping rate is measured with much better accuracy.

\section{CONCLUSION}

Beam-based numerical models of broad-band impedances have been developed at the Diamond Light Source. For each axis (longitudinal, horizontal, and vertical), the model includes a single broad-band resonator and a resistive-wall impedance calculated analytically using known dimensions and resistivity of the vacuum chamber. To get the model parameters, a set of measured data has been used including current-dependent shift of betatron tunes and synchronous phase, chromatic damping times, and bunch lengthening. The turn-by-turn beam position 
was measured using transverse multibunch feedback system, and the longitudinal bunch profile was measured using a streak-camera. Performance of the beam diagnostic equipment was good enough to measure precisely these beam parameters characterizing the interaction between a single-bunch beam and broad-band coupling impedance. A complex frequency shift obtained by solving an eigenvalue problem was used to fit the measured current-dependent betatron tune shift (real part) and chromatic damping rate (imaginary part). This combined fitting technique allows us to improve the fit accuracy of the measured data, especially for the horizontal plane, because the current-dependent shift of the horizontal tune is hardly measurable due to dipole and quadrupole impedance compensation, whereas the damping rate can be measured quite precisely. The beam-based impedance models have been used as input parameters of the multiparticle tracking code, the tracking results have been compared with the measured data. The measured betatron tunes and chromatic damping rates agree rather well with the tracking data. So we can conclude that the impedance models and tracking algorithms are adequate for simulations of single-bunch collective effects resulting from interaction between a beam and the broadband impedance. Since Diamond has the shortest natural bunch length among all light sources in standard operation, the applicability of the collective effects analysis to the regime relative to Diamond can be relevant to many other accelerator facilities including next generation of light sources.

\section{ACKNOWLEDGMENTS}

The authors wish to thank G. Rehm and DLS Diagnostics group for their great help with the measurements.
[1] A. Chao, Physics of Collective Beam Instabilities (Wiley, New York, 1993).

[2] B. W. Zotter and S. A. Kheifets, Impedances and Wakes in High-Energy Particle Accelerator (World Scientific, Singapore, 1998).

[3] W. Bruns, The GdfidL Electromagnetic Field simulator, http://www.gdfidl.del.

[4] Computer Simulation Technology, CST Particle Studio, http://www.cst.com/Products/CSTPS.

[5] B. Podobedov and G. Stupakov, Point-charge wakefield calculations from finite length bunch wake potentials, Phys. Rev. ST Accel. Beams 16, 024401 (2013).

[6] T. Abe et al., in Proceedings of 5th International Particle Accelerator Conference, Dresden, 2014, TUPRIO21 (JACoW, Dresden, Germany, 2014).

[7] M. Klein et al., in Proceedings of the 4th International Particle Accelerator Conference, IPAC-2013, Shanghai, China, 2013 (JACoW, Shanghai, China, 2013), p. TUPWA005.

[8] K. L. F. Bane et al., in Proceedings of the International Particle Accelerator Conference, Kyoto, Japan (ICR, Kyoto, 2010), p. TUPD079.

[9] R. L. Gluckstern, J. van Zeijts, and B. Zotter, Coupling impedance of beam pipes of general cross section, Phys. Rev. E 47, 656 (1993).

[10] N. Nakamura, in Proceedings of 3rd International ERL Workshop, Ithaca, 2009 (JACoW, Ithaca, NY, 2009).

[11] J. Haissinski, Exact longitudinal equilibrium distribution of stored electrons in the presence of self-fields, Il Nuovo Cimento 18, 72 (1973).

[12] B. Zotter, CERN Report No. SPS/81-14 (DI), 1981.

[13] C. Thomas and G. Rehm, Proceedings of the International Particle Accelerator Conference, Kyoto, Japan (ICR, Kyoto, 2010), p. MOPE081.

[14] I. Uzun, M. Abbott, M. T. Heron, A. F. D. Morgan, and G. Rehm, in Proceedings of 13th International Conference on Accelerator and Large Experimental Physics Control Systems, Grenoble, 2011, MOPKSO27 (JACoW, Grenoble, France, 2011). 